\title{
Deviation Prediction and Correction on Low-Cost Atmospheric Pressure Sensors using a Machine-Learning Algorithm
}

\author{
Tiago C. de Araújo ${ }^{1} \mathbb{D}^{\mathrm{a}}$, Lígia T. Silva ${ }^{2} \mathbb{( D}^{\mathrm{b}}$ and Adriano J. C. Moreira ${ }^{1} \mathbb{C}^{\mathrm{c}}$ \\ ${ }^{1}$ Algoritmi Research Centre, Universidade do Minho, Guimarães, Portugal \\ ${ }^{2}$ CTAC Research Centre, Universidade do Minho, Guimarães, Portugal \\ tiago.araujo@ifrn.edu.br,lsilva@civil.uminho.pt,adriano.moreira@algoritmi.uminho.pt
}

\begin{abstract}
Keywords: $\quad$ Low-Cost Sensors, Data Quality, Machine Learning, Environmental Monitoring, Collaborative Sensing.
Abstract: Atmospheric pressure sensors are important devices for several applications, including environment monitoring and indoor positioning tracking systems. This paper proposes a method to enhance the quality of data obtained from low-cost atmospheric pressure sensors using a machine learning algorithm to predict the error behaviour. By using the extremely Randomized Trees algorithm, a model was trained with a reference sensor data for temperature and humidity and with all low-cost sensor datasets that were co-located into an artificial climatic chamber that simulated different climatic situations. Fifteen low-cost environmental sensor units, composed by five different models, were considered. They measure - together - temperature, relative humidity and atmospheric pressure. In the evaluation, three categories of output metrics were considered: raw; trained by the independent sensor data; and trained by the low-cost sensor data. The model trained by the reference sensor was able to reduce the Mean Absolute Error (MAE) between atmospheric pressure sensor pairs by up to $67 \%$, while the same ensemble trained with all low-cost data was able to reduce the MAE by up to $98 \%$. These results suggest that low-cost environmental sensors can be a good asset if their data are properly processed.
\end{abstract}

\section{INTRODUCTION}

Low-cost environmental sensors have enabled individuals to build and manage their own monitoring system not only by its lower price, but also due to its easy availability and extended technical support. Therefore, when engaged individuals share a common concern, such as the quality of the environment, those particular monitoring artefacts, together, can be part of a collaborative monitoring system (Goldman et al., 2009; Zaman et al., 2014). Collaborative sensing, either mobile or not, can be helpful as a complementary tool in several fields of study, such as biology (Kanhere, 2011), urban environment and weather and local climate (D'Hondt et al., 2013; Young et al., 2014).

The use of such sensors can help to reduce overall costs to maintain an urban environmental monitoring system in continuous run. A specific concern about urban areas is the urban heat islands. The continuous

\footnotetext{
a(D) https://orcid.org/0000-0002-1766-5768

b(iD) https://orcid.org/0000-0002-0199-8664

c(iD https://orcid.org/0000-0002-8967-118X
}

monitoring of environmental conditions in urban areas can help in the assessment and in triggering actions towards prevention or mitigation of urban heat islands as demonstrated by (Magli et al., 2016; Qaid et al., 2016; Salata et al., 2017) or other humancaused local phenomena, such as air pollution.

The study of air quality is another field that has also seen an increase in the utilization of low-cost sensors (Kumar et al., 2015). As an example, the authors in (Hu et al., 2016) described the design and evaluation of an air-quality monitoring system that uses low-cost sensors and found the performance of the sensors to be satisfactory; the authors in (Duvall et al., 2016) investigated the performance of low-cost sensors for ozone and nitrogen dioxide monitoring and its application in a community, and found that the sensors, handled by citizen scientists, provided consistent and positive readings in most of the situations. Once the sensors are evaluated positively, they can feed, for example, a local air quality evaluation system as described by (Silva $\&$ Mendes, 
2012). In an indoor scenario, they can also be used for air-quality assessment by monitoring the levels of $\mathrm{CO}_{2}$, once it has relevant consequences on cognitive performance of the occupants, as described by (Allen et al., 2016; Satish et al., 2012). They can even be used as a complementary asset for indoor surveillance (Szczurek et al., 2017).

Electronic sensors are often controlled by hardware with embedded microprocessors, such as Arduino, Raspberry Pi or NodeMCU. Amongst these, Arduino is, perhaps, the most popular among nonspecialized users in a citizen science scope. Several works about Internet of Things, Environmental Monitoring and Sensor Evaluations used Arduino as a platform for data collection due to its ease of use and widespread collaborative support (de Araújo et al., 2017; Fuertes et al., 2015; Piedrahita et al., 2014; Saini et al., 2016; Sinha et al., 2015; Trilles et al., 2015).

However, low-cost environmental sensors cannot be deployed into the field without minimal verifications regarding their data-quality, even when nominally calibrated from factory. Afterall, the value of a running sensing system is strictly related to the quality of its data, as scrutinized by (Liu et al., 2015). Data quality assessment, by itself, is a difficult task, mostly because bad quality data can be originated from diverse sources, including a bad sensor behaviour (Gitzel, 2016). In air quality studies, the investigators in (Borrego et al., 2016) studied the data quality of microsensors by comparison with reference methods for air quality monitoring. They found that the performance can vary from one sensor to another, even if being of the same type. With similar results, authors in (Castell et al., 2017) found that low-cost sensors, despite its issues on reproducibility, can provide very good data for lower-tie applications, such as pollution awareness and environmental monitoring in a coarse scale. However, improvements are necessary if the goal is a high-accuracy application.

Authors in (Terando et al., 2017) and (Ashcroft, 2018) discussed the errors involved in environmental monitoring with microsensors and professional stations. They pointed out that both approaches may not differ in terms of error sources, since biased temperature readings may be common in both situations due to the lack of standardization on thermal shields and positioning. However, despite the technical issues involved in temperature monitoring, the author in (Mwangi, 2017) demonstrated the importance of low-cost sensors for building weather stations in developing countries, places without sufficient resources for conventional monitoring. In the reported experiment, the sensors were first calibrated by placing the low-cost monitoring station close to reference instruments and, then, the artefacts were deployed into the field, achieving good results.

The use of barometric pressure sensors in weather monitoring is important since pressure is a good predictor for rainfall, as it is closely related to water evaporation rate (Özgür \& Koçak, 2015). In simple terms, low pressure values, compared to typical values, may indicate rain, whereas high pressure values may indicate clean weather. Beyond environmental monitoring, atmospheric pressure has also great importance for medical applications, automotive industry and positioning estimation for mobile computing (Yunus et al., 2015). In indoor and outdoor positioning systems, barometric pressure can provide a good estimate of altitude, since the air pressure value is about $1013 \mathrm{hPa}$ at sea-level and, it drops by approximately $0.11 \mathrm{hPa}$ per meter in the first 1000 meters of altitude. Thus, the enhancement of data quality in these sensors may empower its use for several applications.

Machine learning has also attracted the attention of non-specialized users in citizen science projects. Some contributing factors to its spreading are its accessibility through built-in open-source packages, such as "scikit-learn" for Python programming language (Scikit-Learn, 2019), and the available tutorials and collaborative support by other users through web communities such as GitHub, Quora and StackExchange. Machine learning algorithms can be applied to sensor analysis as a powerful calibration tool. Authors in (Yamamoto et al., 2017) used a machine learning-based model for calibration of temperature sensors in outdoor monitoring, and reduced the errors of subject sensors satisfactorily. For air quality applications, the authors in (Zimmerman et al., 2018) used Random Forest ensemble as a regressor between multidimensional data for air-pollutant sensors, including crosssensitivity. They reached the US EPA recommendations for air quality with the calibrated low-cost sensors (US-EPA, 2019), highlighting a promising strategy to overcome poor data, commonly found in low-cost air quality sensors.

\section{RESEARCH PROBLEM AND APPROACH}

The work described in this paper is part of a research project about the use of low-cost environmental sensors for monitoring and characterization of urban 
spaces. Its main concern is the data quality obtained from low-cost sensors.

The most common physical quantities observed in environmental monitoring are air temperature, relative humidity and atmospheric pressure. Sensors involved in the monitoring of these quantities can often have heterogeneous accuracy, with humidity and pressure readings being dependent, at least, on the temperature at which the readings were taken. Therefore, the errors associated to the readings of a sensor might be a function of other parameters.

Regarding the atmospheric pressure sensors, as their transduction principle relies on the surface deformation of the sensing element due to the surrounding air pressure, as described by (MinhDung et al., 2013), it is expected that both temperature and humidity interfere with the sensor readings.

These considerations lead to the hypothesis that the errors, or deviations, from atmospheric pressure sensor readings can be mathematically modeled from temperature and humidity readings.

We propose the use of a supervised machine learning algorithm in the modelling process, as current available regressor algorithms have been shown to be very effective in solving problems using both linear and non-linear approximations. The chosen machine-learning algorithm for the prediction of errors in atmospheric pressure readings was the Extremely Randomized Trees (Extra-trees) ensemble regressor, first proposed by (Geurts et al., 2006), and available in "sklearn" library for Python 3.7. This algorithm is suitable for both linear and non-linear systems with good accuracy and computational efficiency.

In short terms, in the learning process the algorithm requires both input and target vectors, so it can "learn" the behaviour of the output (target) from the given inputs. Once the model is trained, it can either provide outputs using new inputs (data that were not passed in the training process) or correct the whole existing dataset, by feedback, to assess the algorithm's performance in the error prediction.

In the present approach, the input data is composed by the temperature and humidity readings, obtained from a set of sensors co-located and submitted to the same environmental conditions. In the final dataset each individual sensor data is represented by a vector (or feature), that, together, formed a matrix " $m \times n$ ", where " $m$ " is the number of reading samples (the vector length), and " $n$ " is the number of sensors. The target vector was obtained from the deviations between a pair of pressure sensors, point to point, where one of them was considered as a beacon (a reference). Both data were merged and then used in the training process. Consequently, once trained, the model was able to predict the errors between the atmospheric pressure sensors using the temperature and humidity data as input. The predicted error was added back into the original pressure sensor dataset to, finally, obtain its corrected readings.

The overall process is illustrated in Figure 1, in which: " $\mathrm{t}_{1},(\ldots), \mathrm{t}_{\mathrm{N} 1}$ " are vectors containing the temperature readings from " $\mathrm{N}_{1}$ " different sensors; " $\mathrm{h}_{1},(\ldots), \mathrm{h}_{\mathrm{N} 2}$ " are vectors containing the humidity sensor readings from " $\mathrm{N}_{2}$ " different sensors; " $\mathrm{p}_{\text {ref }}$ " is the vector containing the readings from atmospheric pressure sensor used as reference; " $\mathrm{p}_{\mathrm{i}}$ " is the vector containing the readings from a given atmospheric pressure sensor " $\mathrm{i}$ " which will have its readings adjusted; " $\mathrm{y}_{\mathrm{i}}$ " is the actual error between the given sensors (used only in the learning process); " $\hat{y}_{i}$ " is the output vector of the model, containing the predicted error (a function of temperature and humidity); and, finally, " $\hat{p}_{i}$ " is the vector containing the corrected atmospheric pressure sensor readings. All vectors have length " $m$ ".

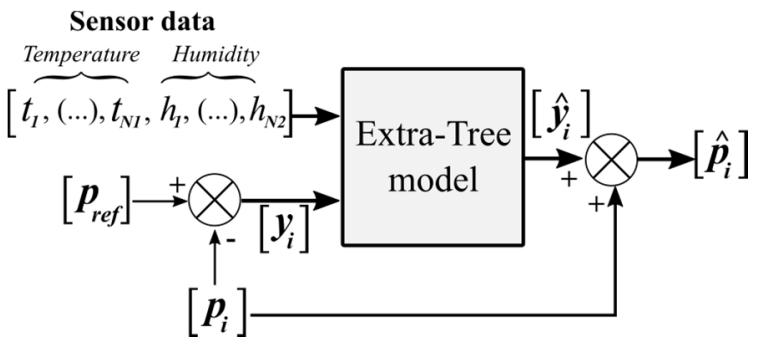

Figure 1: Diagram of the approach adopted to predict and correct deviations between atmospheric pressure sensors.

Moreover, machine-learning algorithms often allow the adjustment of internal parameters that affect its performance. The main adjustable parameters for performance tuning in the Extra-Trees ensemble regressor are the number of trees in the forest and the maximum number of split nodes in the decision trees (this last is used only in classification problems). The choice of the number of trees has a trade-off: higher number of trees results in better accuracy but requires more computational resources. Usually, the default value for this parameter is " 100 " and is often used as a starting value. In case of eventual unsatisfactory performance, it could be increased or decreased in a fine-tuning process.

The next section contains the experimental description that generated the datasets used in the current investigation. 


\section{EXPERIMENTAL}

The datasets used in this study were obtained from sensors submitted to controlled conditions inside an artificial climatic chamber (Aralab Fitoclima ${ }^{\circledR}$ ) that followed a set of instructions to create different temperature and humidity combinations. The following sections describe the preparation of the sensor sets and the experimental execution plan for the artificial climatic chamber.

\subsection{Sensor Selection and Preparation}

Five different models of sensors were chosen due to its price, availability and ease of use (presence of built-in interface): AM2302 (Adafruit, 2016), HTU21D (Measurement Specialties Inc., 2013), BMP180 (Bosch Sensortec, 2013), BME280 (Bosch Sensortec, 2015) and MPL3115A2 (Freescale Semiconductor, 2013) (Figure 2). From these, the BME280 provides temperature, humidity and pressure data; AM2302 and HTU21D provide temperature and humidity data; BMP180 and MPL3115A2 provide temperature and pressure data. Three units of each sensor model were used, forming three sets containing one unit from each sensor model (identified as Set A, Set B and Set C).

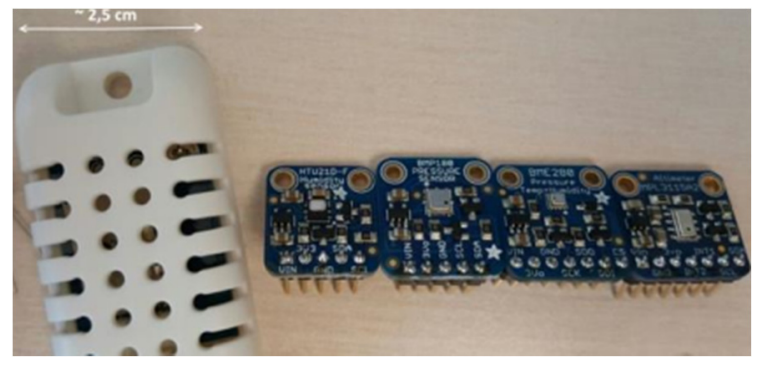

Figure 2: Low-cost sensors selected for the experiment. From left to right: AM2302, HTU21D, BMP180, BME280 and MPL3115A2.

Each experimental set was assembled around one Arduino device, equipped with a "SD\&RTC" shield for datalogging with timestamps. There were no physical relevant spacing between sensors, so they could read the same quantity values. The sampling rate was set to one sample per minute.

An additional independent sensor from Lascar Electronics $\mathbb{C}$ (Lascar Electronics, n.d.), factorycalibrated for temperature and humidity, was also placed together with the low-cost sensor sets inside the chamber. Its function was to provide reference data for later processing and to enable the comparison of the deviation prediction performances between the model trained with all sensors and the model trained only with reference data. Every device was timely synchronized to avoid reading displacements between datasets.

\subsection{Artificial Climatic Chamber Configuration}

Two experimental profiles were programmed into the climatic chamber. The first one was programmed with temperature levels of $-5^{\circ} \mathrm{C}, 10^{\circ} \mathrm{C}, 25^{\circ} \mathrm{C}$ and $40{ }^{\circ} \mathrm{C}$; humidity levels of $30 \%, 50 \%$ and $80 \%$. The total experiment length was 46 hours, including: 11.5 hours for each temperature level in stability; 3 hours for each humidity level in stability. The second experimental profile was set by removing the negative temperature from the first profile and equally extending the times of each steady level. This was performed to increase the stability time of relative humidity and consequently reduce eventual noise in this quantity.

The programmed execution started at the lowest value of temperature and ended at the highest. The relative humidity level sequence followed the scheme presented in Figure 3. Note that, in the used chamber, it was not possible to control relative humidity below $0^{\circ} \mathrm{C}$. The readings during transitions - either temperature or humidity - are also of interest and were considered.

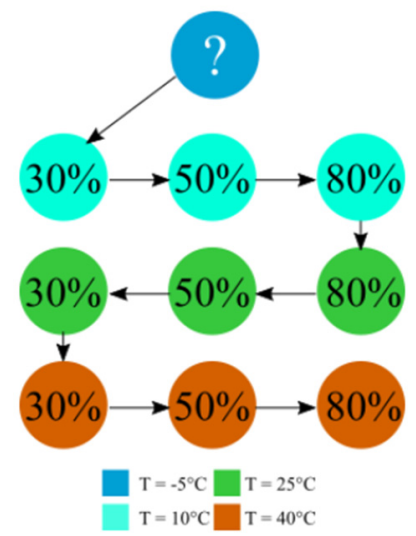

Figure 3: Temperature and humidity sequences programmed into the artificial climatic chamber.

Those levels were chosen to attempt to cover typical conditions of an urban environment that does not experience extreme weather conditions. Consequently, it should allow the assessment of the data quality of the low-cost sensors in short-term timespan within the given hypothetic scenario. After the execution of all the experiments, more than 560 hours of continuous controlled readings of 
temperature and humidity were obtained, as well as corresponding readings of the natural atmospheric pressure (uncontrolled), since the chamber is not sealed.

The final dataset, generated by joining all the data gathered from each experiment, consisted of eleven features (columns) separated by sensor models for the different physical quantities (with no distinction between different sensor sets): five for temperature and three for humidity, used entirely in the training, and three for atmospheric pressure that were used to form the error vectors. Also, the reference provided three complementary features that were used in a separate training: temperature, humidity and dewpoint estimative.

The analysis of the final dataset and respective metrics is described in the next section.

\section{PERFORMANCE METRICS AND DATA ANALYSIS}

As previously described, the target vectors used in the algorithm contained only the errors and were obtained by the deviations point-to-point between two sensor models of the pressure sensors readings by choosing one sensor as beacon: the BME280 (since it was the newest device, among the pressure sensors used). As there were other two models able to provide atmospheric pressure values, it led to two target features vectors: $\boldsymbol{y}_{\boldsymbol{1}}$ and $\boldsymbol{y}_{2}$. The target vector $\boldsymbol{y}_{\boldsymbol{1}}$ was obtained by subtracting the BMP180 pressure readings from BME280 data (1), whilst the target vector $\boldsymbol{y}_{2}$ was obtained by subtracting the MPL3115A2 pressure readings from BME280 data (2), as follows:

$$
\begin{aligned}
& \boldsymbol{y}_{\mathbf{1}}=P_{B M E 280}-P_{B M P 180} \\
& \boldsymbol{y}_{\mathbf{2}}=P_{B M E 280}-P_{M P L 3115 A 2}
\end{aligned}
$$

As each individual sensor, by model and physical quantity measured, was treated as one input feature vector (except the atmospheric pressure readings, that were used only to form the target vectors), it led into eight input vectors, where five input vectors were temperature readings (from AM2302, HTU21D, BMP180, BME280 and MPL3115A2) and three input vectors were relative humidity readings (from AM2302, HTU21D, BME280). The second analysis was performed using the same target vectors generated by the pressure sensors but using only the independent sensor readings as input (temperature, humidity and dew point) for training the model.

In the model preparation stage, the train/test length was set to $80 / 20 \%$ in all analysis, and the number of trees of the network was set to the default value (100). It is a common starting condition, where - nominally - more is better. In case of an eventual poor performance, it could be adjusted later: increased in case of bad metrics; reduced in case of good metrics but with slow training time.

After the training stage with $80 \%$ of the input data (temperature, humidity and pressure deviations), the model was first used to predict the other $20 \%$ of the target data, mainly to check its robustness on the data correction. The second step taken was to use the entire input data to predict all the errors between the pressure sensors. The output errors $\left(\hat{y}_{1}, \hat{y}_{2}\right)$ were added back into its correspondent sensor-deviation data vector (BMP180 and MPL3115) to perform the compensations (equations 3 and 4). Thus, it resulted in five atmospheric pressure vectors: the three original ones (BME280, BMP180 and MPL3115A2), and two containing the new and adjusted values for the BMP180 and the MPL3115A2 sensor models, that were used in the metrics for the performance evaluation.

$$
\begin{aligned}
& \hat{P}_{B M P 180}=P_{B M P 180}+\hat{y}_{1} \\
& \hat{P}_{M P L 3115 A 2}=P_{M P L 3115 A 2}+\hat{y}_{2}
\end{aligned}
$$

Regarding the metrics, the evaluation of the expected and predicted errors from the sub-datasets originated in the train-test process (the $80 \% / 20 \%$ split on the dataset) were named with "TT" suffix, whilst the datasets generated by feedbacking the predicted error into the whole dataset were named with "FIT" suffix. The metrics considered in the evaluation were the Mean Absolute Error (MAE), the Mean Squared Error (MSE) and the Root Mean Squared Error (RMSE), which are respectively described by the equations (5-7):

$$
\begin{aligned}
& \boldsymbol{M A E}=\frac{1}{n} \sum_{i=1}^{n}\left|y_{i}-\hat{y}_{i}\right| \\
& \boldsymbol{M S E}=\frac{1}{n} \sum_{i=1}^{n}\left(y_{i}-\hat{y}_{i}\right)^{2} \\
& \boldsymbol{R M S E}=\sqrt{\frac{1}{n} \sum_{i=1}^{n}\left(y_{i}-\hat{y}_{i}\right)^{2}}
\end{aligned}
$$


These metrics were calculated using different datasets as follows: between original atmospheric pressure vectors, identified as "Raw"; between train and test sub-datasets that used low-cost sensor data for training, identified as " $L C \_T T$ "; between train and test sub-datasets that used independent sensor data for training, identified as "IS_TT"; between deviationcompensated vectors that used low-cost sensor data for training, identified as "LC_FIT"; and between deviation-compensated vectors that used independent sensor readings for training, identified as "IS_FIT". To complement the analysis, the determination coefficient $\left(r^{2}\right)$ and Spearman's Rank-Order Correlation Coefficient $(\rho)$ were also obtained from the described datasets.

\section{RESULTS}

The presented numeric metrics were obtained by averaging ten executions of the Extremely Randomized Forests ensemble regressor with the "shuffle" feature enabled in each train/test split process (cross-validation). This setup ensured that the algorithm was trained and tested with different datapoints in each execution. Table 1 presents the achieved results in numerical terms. The datasets are identified as aforementioned.

There was no significant discrepancy for mean absolute errors (MAE) in raw data between the pairs of sensors: MAE between BME280 and BMP180 is $0.7935 \mathrm{hPa}$, and between BME280 and MPL3115A2 is $0.7958 \mathrm{hPa}$. When the input data of the machinelearning algorithm is only the independent sensor data, containing temperature, humidity and dew point estimated values, the overall MAE was reduced by $68 \%$ for BMP 180 and by $26 \%$ for MPL3115A2, reaching $0.248 \mathrm{hPa}$ and $0.5872 \mathrm{hPa}$, respectively. However, when all low-cost sensor readings were considered for training the model, the error was significantly reduced: the MAE between BME280 and BMP 180 was reduced by $98.6 \%$, while between BME280 and MPL3115A2 was reduced by $98.9 \%$, reaching $0.0109 \mathrm{hPa}$ and $0.0086 \mathrm{hPa}$, respectively.

Table 1: Numerical performance summary (hPa) obtained from different dataset categories.

\begin{tabular}{|c|c|c|c|}
\hline DatasetBME vs. & MAE & MSE & RMSE \\
\hline \multirow{2}{*}{$\begin{array}{c}\text { BMP180 } \\
\text { Raw }_{\text {MPL3115A2 }}\end{array}$} & 0.7935 & 0.9477 & 0.9735 \\
\hline & 0.7958 & 1.0319 & 1.0158 \\
\hline \multirow{2}{*}{ IS_TT ${ }_{\text {MPL3115A2 }}^{\text {BMP180 }}$} & 0.2583 & 0.1023 & 0.3198 \\
\hline & 0.6133 & 0.5274 & 0.7262 \\
\hline \multirow{2}{*}{ IS_FIT ${ }_{\text {MPL3 } 3115 \mathrm{~A} 2}$} & 0.2480 & 0.0936 & 0.3059 \\
\hline & 0.5872 & 0.4794 & 0.6924 \\
\hline \multirow{2}{*}{$\mathrm{LC}_{-} \mathrm{TT}_{\text {MPL3115A2 }}^{\text {BMP180 }}$} & 0.0540 & 0.0061 & 0.0780 \\
\hline & 0.0427 & 0.0063 & 0.0793 \\
\hline \multirow{2}{*}{ LC_FIT $_{\text {MPL3115A2 }}^{\text {BMP180 }}$} & 0.0109 & 0.0012 & 0.0349 \\
\hline & 0.0086 & 0.0013 & 0.0355 \\
\hline
\end{tabular}

Figure 4 shows the timeseries plots containing raw readings, the compensated values using low-cost sensor datasets and the compensated values using independent sensor readings. It is possible to observe that there were no significant deviations in data adjusted by the model that considered all low-cost sensor data. The abrupt changes visible in the timeseries plot was caused by appending subsequent experimental data when the last value of the previous experiment was different from the first value of the subsequent one. As these anomalies occurs once after every $2000+$ samples, it does not cause significant depreciation in numeric performance and is of no concern.

On the contrary of the model that used all sensors, the adjusted data that used only the independent

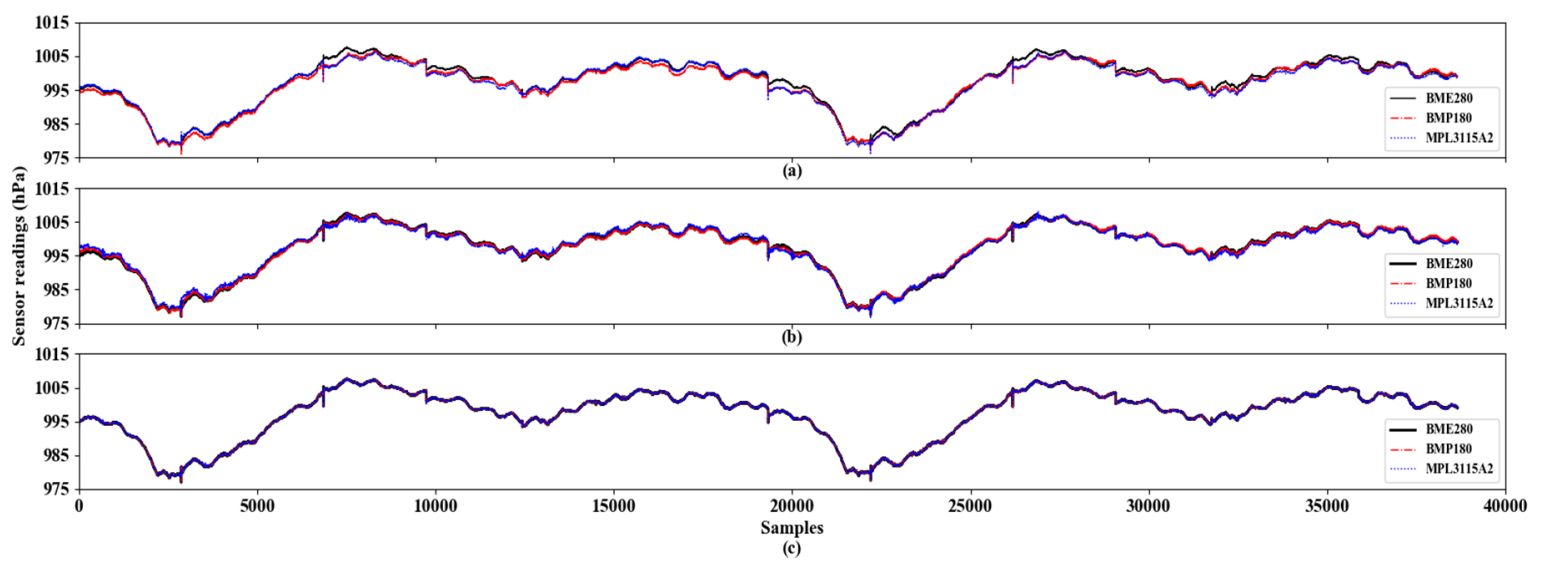

Figure 4: Timeseries of atmospheric pressure readings: (a) raw data; (b) data adjusted by the model trained with the independent sensor data; (c) data adjusted by the model trained with the low-cost weather sensors data. 
sensor readings as input presented deviations that can be visually detected at some points, suggesting that the reduced number of input features, even from a certified sensor, may not reach the same efficacy, for this purpose, as a set of low-cost sensors co-located.

The plot containing the error scattering between raw vectors is presented in Figure 5, and the residuals plot (difference between predicted and actual deviations) of the model trained with the independent sensor and the model trained with all sensor data are presented in Figures 6 and 7, respectively.
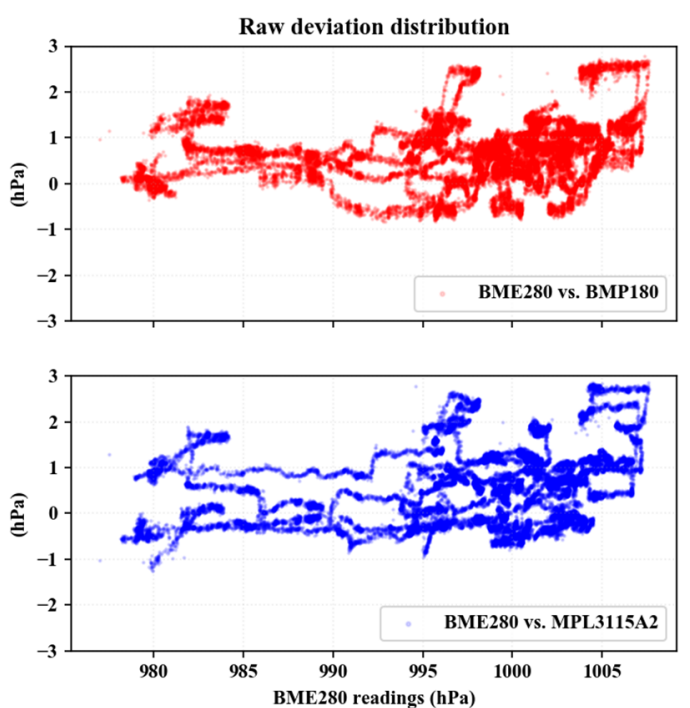

Figure 5: Deviation distribution of the atmospheric pressure sensor readings as obtained (raw).

The density of the error scattering can be interpreted by the colour intensity: faint colours corresponds to low concentration of deviations; vivid and solid colours corresponds to higher concentration of deviations.

An ideal behaviour of the residuals distribution in a fitted model would be a straight horizontal line on zero $\mathrm{hPa}$. The residuals plot of the model trained with the independent sensor ("IS_FIT") indicates that there was still a random behaviour of the deviations. However, it achieved a better performance for BMP180 than for MPL3115A2, as shown by comparing Figures 5 and 6.

The residuals scatterplot of the model trained with all co-located low-cost sensor data ("LC_FIT") presents a very low spread around zero: a good approximation to the ideal behaviour for a fitted model.

The positive results obtained by the Extremely Random Trees algorithm, when using readings from all co-located low-cost sensors, can be extended to the correction of outliers. Although it is still possible to perceive the presence of few outliers in Figure 7 (the vertically spaced points around $983 \mathrm{hPa}$ and $995 \mathrm{hPa}$ ), they were significantly reduced if compared to the "IS FIT" model residuals. Based on these observations, it can be concluded that the "LC_FIT" managed to notoriously reduce the readings deviations.
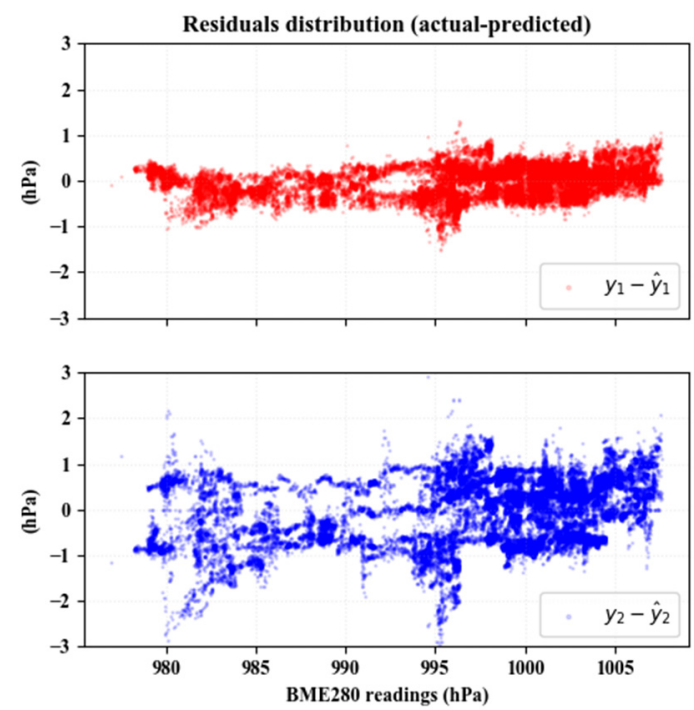

Figure 6: Residuals distribution of Extremely Random Trees ensemble regressor trained with temperature, humidity and dew point estimative from independent sensor ("IS_FIT").
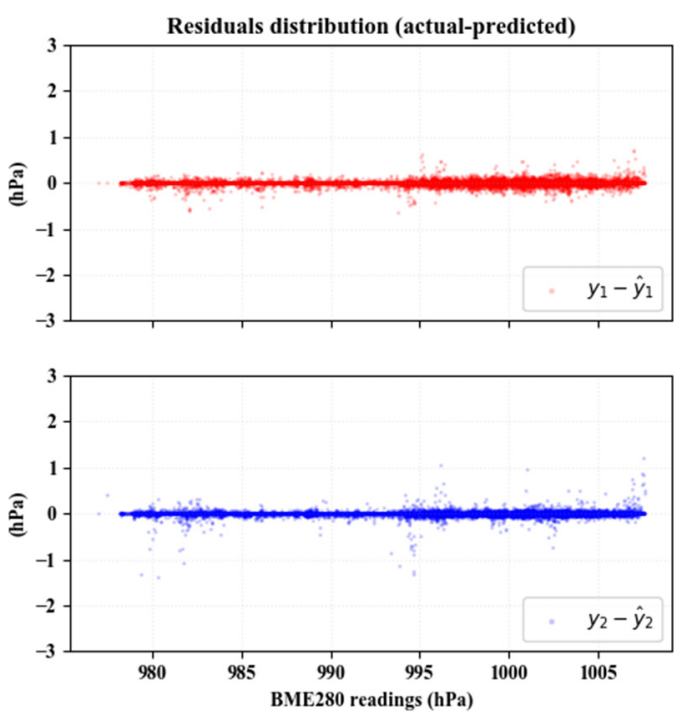

Figure 7: Residuals distribution of Extremely Random Trees ensemble regressor trained with temperature and humidity from co-located low-cost sensors ("LC_FIT").

The determination coefficients $\left(r^{2}\right)$ and Spearman's rank-order correlation coefficient $(\rho)$ of 
the datasets, before and after the Extremely Random Tree algorithm fitting, are exposed in Table 2. The lowest $r^{2}$ was observed between BME280 and MPL3115A2 in raw datasets, with a value of 0.9798; the highest $r^{2}$ was observed in "LC FIT" datasets, with both vectors exceeding the 0.9999 value when compared to BME280. The lowest $\rho$ was also observed between BME280 and MPL3115A2 in raw datasets, with a value of 0.9861 , and the highest $\rho$ was also observed in "LC_FIT" datasets, with both vectors exceeding the value of 0.9999 .

Table 2: Determination coefficients $\left(r^{2}\right)$ and Spearman's rank-order correlation coefficient $(\rho)$ between atmospheric pressure sensor readings before and after deviation compensation by the machine-learning model.

\begin{tabular}{clcc}
\hline \multirow{2}{*}{ Dataset } & & \multicolumn{2}{c}{ BME280 versus: } \\
& & BMP180 & MPL3115A2 \\
\hline \multirow{2}{*}{ "Raw" } & $r^{2}$ & 0.9815 & 0.9798 \\
& $\rho$ & 0.9909 & 0.9861 \\
\hline \multirow{2}{*}{ "IS_FIT" } & $r^{2}$ & 0.9982 & 0.9905 \\
& $\rho$ & 0.9982 & 0.9913 \\
\hline \multirow{2}{*}{ "LC_FIT" } & $r^{2}$ & $0.9999+$ & $0.9999+$ \\
& $\rho$ & $0.9999+$ & $0.9999+$
\end{tabular}

To enrich the interpretation on how the model managed to predict the errors, it is relevant to analyse the feature importance plot. This information can be obtained from the Extremely Randomized Forest model. It reveals which features had an informative role during the training of the model and which features do not inflict significative influence in the outputs. In other words, and for this case, it permits the determination of which data the error of evaluated atmospheric pressure sensors depends on. The feature importance plot for the model trained with the independent sensor ("IS_FIT") is presented in Figure 8 , whilst the feature importance plot of low-cost trained model ("LC_FIT") is presented in Figure 9.

The interpretation of Figure 8 points out that the BMP180 sensor deviations were mostly temperature dependent $(87 \%$ of importance), while the MPL3115A2 deviations demonstrated to suffer higher, yet small, influence from relative humidity (14\% of importance, versus $2.5 \%$ for BMP 180 ). The dewpoint, calculated by a non-linear formula that considers both temperature and humidity, presented relevant importance for MPL3115 deviation predictions (above $20 \%$ of importance) while it had no significant impact on BMP180 deviation predictions.

Regarding the quantities dependence, the model trained with low-cost sensors (Figure 9) agreed with the information presented by the model trained with the independent sensor: the sum of temperature sensors importance resulted in $93 \%$ for the BMP 180 , and humidity has no informative role (7\%). Meanwhile, the sum of humidity data importance results in $20 \%$ for MPL3115A2, suggesting that this variable may be considered for this sensor calibration

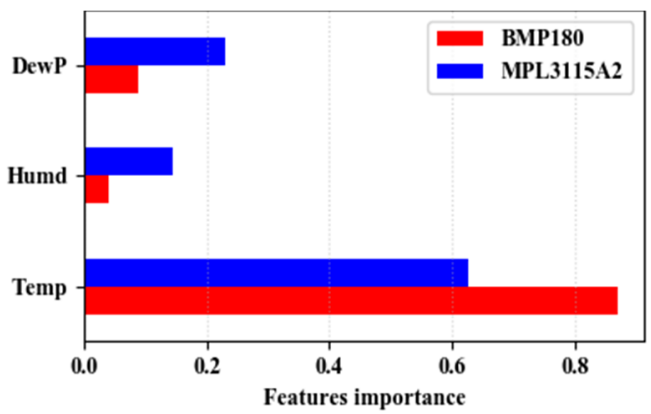

Figure 8: Features importance plot of input vectors used for training the IS_FIT model.

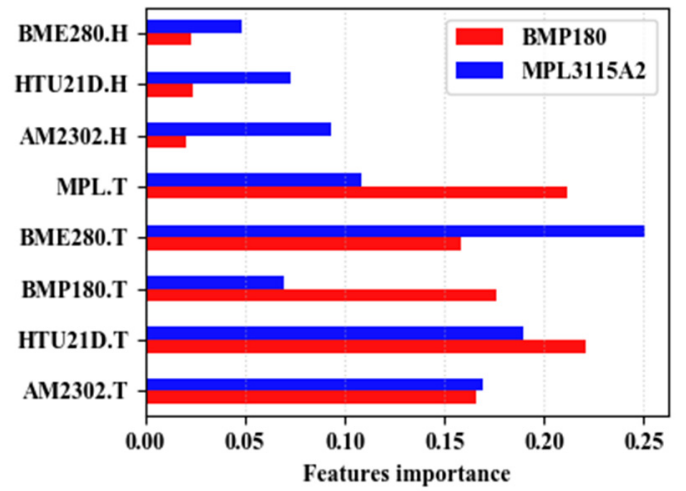

Figure 9: Features importance plot of input vectors used for training the LC_FIT model.

As achieved results were considered positive, no additional hyper-parameter tuning was performed in the machine-learning model. However, it is not discarded that a fine tuning could enhance the algorithm's performance.

\section{DISCUSSION AND CONCLUSIONS}

In this paper, the utilization of a set of co-located lowcost sensors with post-processing in an accessible machine-learning algorithm showed promising results for atmospheric pressure sensor error prediction, once the deviations were reduced by more than $90 \%$ for both sensor models. The achieved reduction on the observed deviations between the sensors, and the consequent data quality 
enhancement, through the utilization of machine learning algorithms is in agreement with the studies presented by (Yamamoto et al., 2017) and (Zimmerman et al., 2018), which used machine learning resources to improve the data quality from temperature and air quality sensors, respectively.

Despite the model trained with the independent sensor was able to reduce deviations between the sensor pairs in acceptable levels, it still did not reach the same performance as the model that learned the error behaviour using the entire low-cost sensors dataset. In a broader reasoning, the low-cost of a given sensor might imply a trade-off on its data quality, but the presented results point out that, if a group of low-cost sensors is used and its data is handled properly (e.g. synchrony, logging and data treatment), the deviation prediction process, and its correction, may be more effective than when just one certified sensor is used. This observation also corroborates with one of the main points of the collaborative sensing that is spreading the low-cost sensor units (fixed or mobile) to overcome the quality of a singular sensing node. Then, it may act as a complementary asset to help conventional methods to address a problem, or even be a palliative in certain situations (Giordano \& Puccinelli, 2015). An example that illustrates the low-cost sensors playing informative role in places where conventional methods are not available yet in large scale is the project with low-cost weather stations for developing countries described by (Mwangi, 2017), that used the HTU21D and BMP180 sensors - both used in this paper. The stations were tested in NOAA facilities and then deployed into field in Kenya for continuous weather monitoring in remote places. Such work can expand into an enhanced environmental sensor network involving the "low-cost" concept, but with "certified" results, similar to the work presented by (Ingelrest et al., 2010).

Regarding the analysis, the most intuitive example of potential application from the observed outcomes is a real time calibration service using a previously trained machine-learning model. Although the model was trained with offline data, after the learning process, it can be executed online, in real time. This is possible to be done by performing the object serialization (or model persistence) in Python programming language, for example. In short, it allows the serialization (export) of an offline trained object (e.g. the trained Extremely Random Trees ensemble regressor used in this work) into a stream of bytes and performs its portability (import) to other service, such as an online server, or a middleware, similar to the author's proposals about data quality improvements in (Dua et al., 2009; Fersi, 2015).

It should be highlighted that the presented results were obtained by considering the BME280 as a beacon (a non-certified reference). However, these results do not show any evidence that they could not be replicated and reach similar positive performance if the target vectors $\left(y_{1}\right.$ and $\left.y_{2}\right)$ were obtained from a reference sensor for atmospheric pressure instead of a beacon sensor, since the machine learning is able to predict the error behaviour regardless of the number of inputs. Although the environmental dependence of the error between atmospheric pressure sensor were expected, since the considered physical quantities are strictly related one to another, the Extra-Trees algorithm would manage to pick only the features that relevantly can describe the observed problem. In other words, if the input had more, and even irrelevant, features (e.g. timestamp, luminosity, etc), the obtained results would not be different.

From this point on, some possibilities of subsequent works can be considered, such as: a field test (uncontrolled conditions) of the error prediction using the approach of this work, and the consequent evaluation of its robustness for long-term sensor use, or its robustness over sensor positioning (spatial variation); the investigation of the performance of this approach when using a certified reference sensor for atmospheric pressure as the generator for the target vectors (instead of a beacon sensor, as aforementioned); to assess the performance of different machine learning algorithms for offline and online sensor correction (e.g. Random Forests, SVM regressor, Lasso, etc.) and different hyper parameters tuning.

Finally, it is expected that the presented work, its respective results, and the opened opportunities may provide contributions or further motivations for studies situated in the intersection zone between citizen science, big data and environmental awareness and monitoring, or even those beyond these areas but which objectives eventually include the enhancement of data quality from environmental microsensors.

\section{REFERENCES}

Adafruit. (2016). AM2302/DHT22 Datasheet (pp. 1-5). pp. 1-5. Retrieved from https://cdnshop.adafruit.com/datasheets/Digital+humidity + and $+\mathrm{t}$ emperature+sensor+AM2302.pdf

Allen, J. G., MacNaughton, P., Satish, U., Santanam, S., Vallarino, J., \& Spengler, J. D. (2016). Associations of cognitive function scores with carbon dioxide, ventilation, and volatile organic compound exposures 
in office workers: A controlled exposure study of green and conventional office environments. Environmental Health Perspectives, 124(6), 805-812. https://doi.org/10.1289/ehp.1510037

Ashcroft, M. B. (2018). Which is more biased: Standardized weather stations or microclimatic sensors? Ecology and Evolution, 5231-5232. https://doi.org/10.1002/ece3.3965

Borrego, C., Costa, A. M., Ginja, J., Amorim, M., Coutinho, M., Karatzas, K., ... Penza, M. (2016). Assessment of air quality microsensors versus reference methods: The EuNetAir joint exercise. Atmospheric Environment, 147(2), 246-263. https://doi.org/10.1016/j.atmosenv.2016.09.050

Bosch Sensortec. (2013). BMP180 Datasheet (p. 28). p. 28. Retrieved from https://cdnshop.adafruit.com/datasheets/BST-BMP180-DS00009.pdf

Bosch Sensortec. (2015). BME280 Datasheet. Retrieved from http://www.boschsensortec.com/en/homepage/products_3/environmenta 1_sensors_1/bme280/bme280_1

Castell, N., Dauge, F. R., Schneider, P., Vogt, M., Lerner, U., Fishbain, B., ... Bartonova, A. (2017). Can commercial low-cost sensor platforms contribute to air quality monitoring and exposure estimates? Environment International, 99, 293-302. https://doi.org/10.1016/j.envint.2016.12.007

D'Hondt, E., Stevens, M., \& Jacobs, A. (2013). Participatory noise mapping works! An evaluation of participatory sensing as an alternative to standard techniques for environmental monitoring. Pervasive and Mobile Computing, 9(5), 681-694. https://doi.org/10.1016/j.pmcj.2012.09.002

de Araújo, T. C., Silva, L. T., \& Moreira, A. C. (2017). Data Quality Issues on Environmental Sensing with Smartphones. Proceedings of the 6th International Conference on Sensor Networks: SENSORNETS., 5968. https://doi.org/10.5220/0006201600590068

Dua, A., Bulusu, N., \& Feng, W. (2009). Towards Trustworthy Participatory Sensing. 4th USENIX Workshop on Hot Topics in Security (HotSec-09).

Duvall, R., Long, R., Beaver, M., Kronmiller, K., Wheeler, M., \& Szykman, J. (2016). Performance Evaluation and Community Application of Low-Cost Sensors for Ozone and Nitrogen Dioxide. Sensors, 16(10), 1698. https://doi.org/10.3390/s16101698

Fersi, G. (2015). Middleware for internet of things: A study. Proceedings - IEEE International Conference on Distributed Computing in Sensor Systems, DCOSS 2015, 2(3), 230-235. https://doi.org/10.1109/DCOSS.2015.43

Freescale Semiconductor. (2013). MPL3115A2 Datasheet. Retrieved from https://cdnshop.adafruit.com/datasheets/1893_datasheet.pdf

Fuertes, W., Carrera, D., Villacis, $\bar{C}$., Toulkeridis, T., Galarraga, F., Torres, E., \& Aules, H. (2015). Distributed System as Internet of Things for a New Low-Cost, Air Pollution Wireless Monitoring on Real Time. 2015 IEEE/ACM 19th International Symposium on Distributed Simulation and Real Time Applications (DS-RT), 58-67. https://doi.org/10.1109/DSRT.2015.28

Geurts, P., Ernst, D., \& Wehenkel, L. (2006). Extremely randomized trees. Machine Learning, 63(1), 3-42. https://doi.org/10.1007/s10994-006-6226-1

Giordano, S., \& Puccinelli, D. (2015). When sensing goes pervasive. Pervasive and Mobile Computing, 17(PB), 175-183. https://doi.org/10.1016/j.pmcj.2014.09.008

Gitzel, R. (2016). Data quality in time series data: An experience report. CEUR Workshop Proceedings, 1753, 41-49.

Goldman, J., Shilton, K., Burke, J., Estrin, D., Hansen, M., Ramanathan, N., ... Samanta, V. (2009). Participatory Sensing - A Citizen-powered approach to illuminating the patterns that shape our world. Los Angeles, California, USA: Center for Embedded Networked Sensing.

Hu, K., Sivaraman, V., Luxan, B. G., \& Rahman, A. (2016). Design and Evaluation of a Metropolitan Air Pollution Sensing System. IEEE Sensors Journal, 16(5), 14481459 .

Ingelrest, F., Barrenetxea, G., Schaefer, G., Vetterli, M., Couach, O., \& Parlange, M. (2010). Sensorscope: Application-Specific Sensor Network for Environmental Monitoring. ACM Transactions on Sensor Networks, 6(2), 1-32. https://doi.org/10.1145/1689239.1689247

Kanhere, S. S. (2011). Participatory Sensing: Crowdsourcing Data from Mobile Smartphones in Urban Spaces. 2011 IEEE 12th International Conference on Mobile Data Management, 3-6. https://doi.org/10.1109/MDM.2011.16

Kumar, P., Morawska, L., Martani, C., Biskos, G., Neophytou, M., Di Sabatino, S., ... Britter, R. (2015). The Rise of Low-Cost Sensing for Managing Air Pollution in Cities. Environment International, 75, 199-205. https://doi.org/10.1016/j.envint.2014.11.019

Lascar Electronics. (n.d.). Certificate of Calibration. Retrieved June 28, 2016, from $\mathrm{http} / /$ www.lascarelectronics.com/pdf-usbdatalogging/data-logger0800188001331301358.pdf

Liu, L., Wei, W., Zhao, D., \& Ma, H. (2015). Urban Resolution: New Metric for Measuring the Quality of Urban Sensing. IEEE Transactions on Mobile Computing, 14(12), 2560-2575. https://doi.org/10.1109/TMC.2015.2404786

Magli, S. ., Lodi, C. ., Contini, F. M. ., Muscio, A. ., \& Tartarini, P. . (2016). Dynamic analysis of the heat released by tertiary buildings and the effects of urban heat island mitigation strategies. Energy and Buildings, 114 , 164-172. https://doi.org/10.1016/j.enbuild.2015.05.037

Measurement Specialties Inc. (2013). HTU21D Datasheet (pp. 1-21). pp. 1-21. Retrieved from https://cdnshop.adafruit.com/datasheets/1899_HTU21D.pdf

Minh-Dung, N., Takahashi, H., Uchiyama, T., Matsumoto, K., \& Shimoyama, I. (2013). A barometric pressure sensor based on the air-gap scale effect in a cantilever. 
Applied Physics Letters, 103(14), 103-106. https://doi.org/10.1063/1.4824027

Mwangi, C. (2017). Low Cost Weather Stations for Developing Countries (Kenya). 7th United Nations International Conference on Space-Based Technologies for Disaster Risk Reduction, (October). Retrieved from http://www.unspider.org/sites/default/files/21. UNSPIDER Presentation - Mwangi.pdf

Özgür, E., \& Koçak, K. (2015). The effects of the atmospheric pressure on evaporation. Acta Geobalcanica, 1(1), 17-24. https://doi.org/10.18509/agb.2015.02

Piedrahita, R., Xiang, Y., Masson, N., Ortega, J., Collier, A., Jiang, Y., ... Shang, L. (2014). The next generation of low-cost personal air quality sensors for quantitative exposure monitoring. Atmospheric Measurement Techniques, 7(10), 3325-3336. https://doi.org/10.5194/amt-7-3325-2014

Qaid, A., Bin Lamit, H., Ossen, D. R., \& Raja Shahminan, R. N. (2016). Urban heat island and thermal comfort conditions at micro-climate scale in a tropical planned city. Energy and Buildings, 133, 577-595. https://doi.org/10.1016/j.enbuild.2016.10.006

Saini, H., Thakur, A., Ahuja, S., Sabharwal, N., \& Kumar, N. (2016). Arduino based automatic wireless weather station with remote graphical application and alerts. $3 r d$ International Conference on Signal Processing and Integrated Networks, SPIN 2016, 605-609. https://doi.org/10.1109/SPIN.2016.7566768

Salata, F., Golasi, I., Petitti, D., Vollaro, E. de L., Coppi, M., \& Vollaro, A. de L. (2017). Relating microclimate, human thermal comfort and health during heat waves: an analysis of heat island mitigation strategies through a case study in an urban outdoor environment. Sustainable Cities and Society, 30, 79-96. https://doi.org/10.1016/j.scs.2017.01.006

Satish, U., Mendell, M. J., Shekhar, K., Hotchi, T., Sullivan, D., Streufert, S., \& Fisk, W. J. (2012). Is CO2 an indoor pollutant? direct effects of low-to-moderate $\mathrm{CO} 2$ concentrations on human decision-making performance. Environmental Health Perspectives, $120(12)$, 1671-1677. https://doi.org/10.1289/ehp.1104789

Scikit-Learn. (2019). Scikit-Learn. Retrieved from https://scikit-learn.org/stable/

Silva, L. T., \& Mendes, J. F. G. (2012). City Noise-Air: An environmental quality index for cities. Sustainable Cities and Society, 4(1), 1-11. https://doi.org/10.1016/j.scs.2012.03.001

Sinha, N., Pujitha, K. E., \& Alex, J. S. R. (2015). Xively based sensing and monitoring system for IoT. 2015 International Conference on Computer Communication and Informatics, ICCCI 2015, 8-13. https://doi.org/10.1109/ICCCI.2015.7218144

Sivaraman, V., Carrapetta, J., Hu, K., \& Luxan, B. G. (2013). HazeWatch: A participatory sensor system for monitoring air pollution in Sydney. 38th Annual IEEE Conference on Local Computer Networks - Workshops, 56-64. https://doi.org/10.1109/LCNW.2013.6758498
Szczurek, A., Maciejewska, M., \& Pietrucha, T. (2017). Occupancy detection using gas sensors. SENSORNETS 2017 - Proceedings of the 6th International Conference on Sensor Networks, 2017-Janua(Sensornets), 99-107.

Terando, A. J., Youngsteadt, E., Meineke, E. K., \& Prado, S. G. (2017). Ad hoc instrumentation methods in ecological studies produce highly biased temperature measurements. Ecology and Evolution, 7(23), 98909904. https://doi.org/10.1002/ece3.3499

Trilles, S., Luján, A., Belmonte, Ó., Montoliu, R., TorresSospedra, J., \& Huerta, J. (2015). SEnviro: A sensorized platform proposal using open hardware and open standards. Sensors (Switzerland), 15(3), 55555582. https://doi.org/10.3390/s150305555

US-EPA. (2019). US EPA. Retrieved from https://www.epa.gov/environmental-topics/air-topics

Yamamoto, K., Togami, T., Yamaguchi, N., \& Ninomiya, S. (2017). Machine learning-based calibration of lowcost air temperature sensors using environmental data. Sensors (Switzerland), 17(6), 1-16. https://doi.org/10.3390/s17061290

Young, D. T., Chapman, L., Muller, C. L., Cai, X.-M., \& Grimmond, C. S. B. (2014). A Low-Cost Wireless Temperature Sensor: Evaluation for Use in Environmental Monitoring Applications. Journal of Atmospheric and Oceanic Technology, 31(4), 140320111908003. https://doi.org/10.1175/JTECH-D13-00217.1

Yunus, N., Halin, I., Sulaiman, N., Ismail, N., \& Ong, K. (2015). Valuation on MEMS pressure sensors and device applications. International Journal of Electrical, Computer, Energetic, Electronic and Communication Engineering, 9(8), 768-776.

Zaman, J., D’Hondt, E., Boix, E. G., Philips, E., Kambona, K., \& De Meuter, W. (2014). Citizen-friendly participatory campaign support. 2014 IEEE International Conference on Pervasive Computing and Communication Workshops (PERCOM WORKSHOPS), 232-235. https://doi.org/10.1109/PerComW.2014.6815208

Zimmerman, N., Presto, A. A., Kumar, S. P. N., Gu, J., Hauryliuk, A., Robinson, E. S., ... Subramanian, R. (2018). A machine learning calibration model using random forests to improve sensor performance for lower-cost air quality monitoring. Atmospheric Measurement Techniques, 11(1), 291-313. https://doi.org/10.5194/amt-11-291-2018

\section{APPENDIX}

The datasets used in this work are available in the Zenodo repository, with digital identifier (DOI) as 10.5281/zenodo.3560299.

We encourage the readers to reproduce our findings. 